\title{
General Equations of Motion for a Damaged Asymmetric Aircraft
}

\author{
Barton J. Bacon ${ }^{*}$ and Irene M. Gregory ${ }^{\dagger}$ \\ NASA Langley Research Center, Hampton, VA, 23681
}

\begin{abstract}
There is a renewed interest in dynamic characteristics of damaged aircraft both in order to assess survivability and to develop control laws to enhance survivability. This paper presents a set of flight dynamics equations of motion for a rigid body not necessarily referenced to the body's center of mass. Such equations can be used when the body loses a portion of its mass and it is desired to track the motion of the body's previous center of mass/reference frame now that the mass center has moved to a new position. Furthermore, results for equations presented in this paper and equations in standard aircraft simulations are compared for a scenario involving a generic transport aircraft configuration subject to wing damage.
\end{abstract}

\section{Introduction}

There is a renewed interest in control of aircraft that have sustained damage. This interest is driven by diverse factors such as advances in adaptive flight control, threats from shoulder-fired missiles, mid-air collisions, aging aircraft, and undetermined lifecycles of new composite transport aircraft. Illustrative examples are shown in figure 1. In order to analyze the dynamics of damaged aircraft the dynamic equations of motion must properly reflect the underlying physics. This is not a novel problem from the dynamic perspective. Since the first payloads have been delivered from aircraft in flight, the equations of motion describing these dynamics have been derived from first principles. ${ }^{1}$ With the renewed interest in the problem, the authors hope to provide a convenient reference for the dynamic equations of motion that properly account for non-collocation of the center of mass and the body axes reference point.

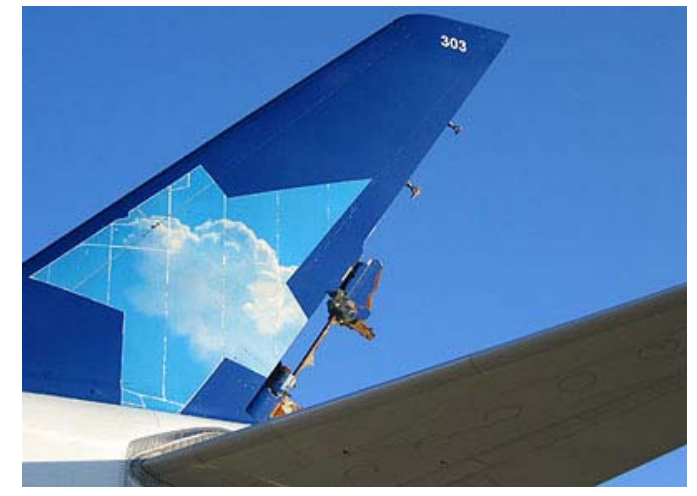

(a)

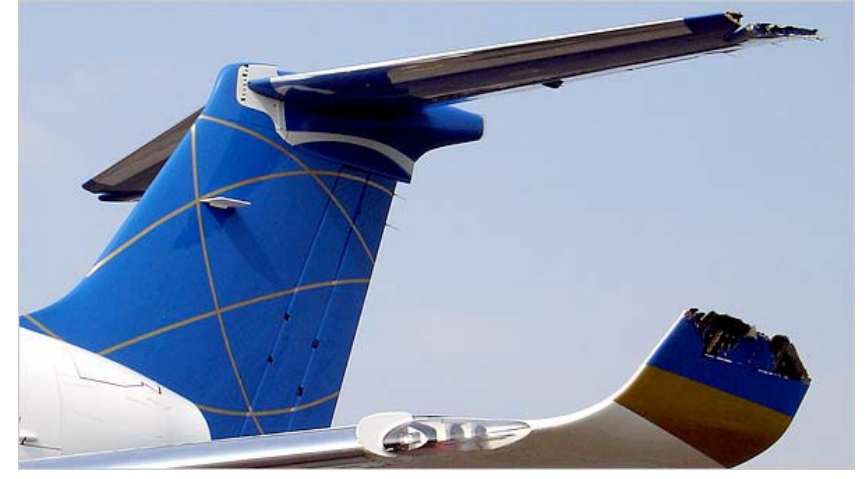

(b)

Figure 1 (a) Air Transat A310 - 2005 - Rudder Loss; (b) Embraer Legacy 600 - Brazil - Wing Tip and Stabilator Loss, September 2006.

To provide a clear exposition, the paper is organized as follows. First, rigid body equations of motion are developed that are not necessarily referenced to the body's center of mass. These equations govern the motion not of the center of mass but some other selected fixed point on the body. One could, for example, place the equation of motion reference at the aerodynamic reference center and automatically obtain the velocity components required to

\footnotetext{
* Senior Aerospace Engineer, Dynamic Systems and Control Branch, NASA LaRC, MS 308, Hampton, VA 23681.

${ }^{\dagger}$ Senior Aerospace Engineer, Dynamic Systems and Control Branch, NASA LaRC, MS 308, Hampton, VA 23681, and AIAA Senior Member.
} 
define angle of attack and sideslip angle, independent variables for the aerodynamic forces and moments. The proposed equations of motion are then applied to the problem of modeling large instantaneous shifts in center of mass of a rotating body that suddenly loses a portion of its mass. Benefits in simulation implementation are discussed. As an example, open loop dynamic responses of a generic transport aircraft with wing damage are compared for typical equations of motion used in the aircraft simulation and the equations presented here.

\section{Rigid Body Equations of Motion Referenced to an Arbitrary Fixed Point on the Body}

There are several approaches that can be used to develop the general equations of motion. The one selected here starts with Newton's laws applied to a collection of particles defining the rigid body (any number of dynamics or physics books can serve as references, e.g. reference 2). In this paper, the rigid body equations of motion over a flat non-rotating earth are developed that are not necessarily referenced to the body's center of mass. Such equations will be used in the next section when the body loses a portion of its mass and it is desired to track the motion of the body's previous center of mass/reference frame now that the mass center has moved to a new position

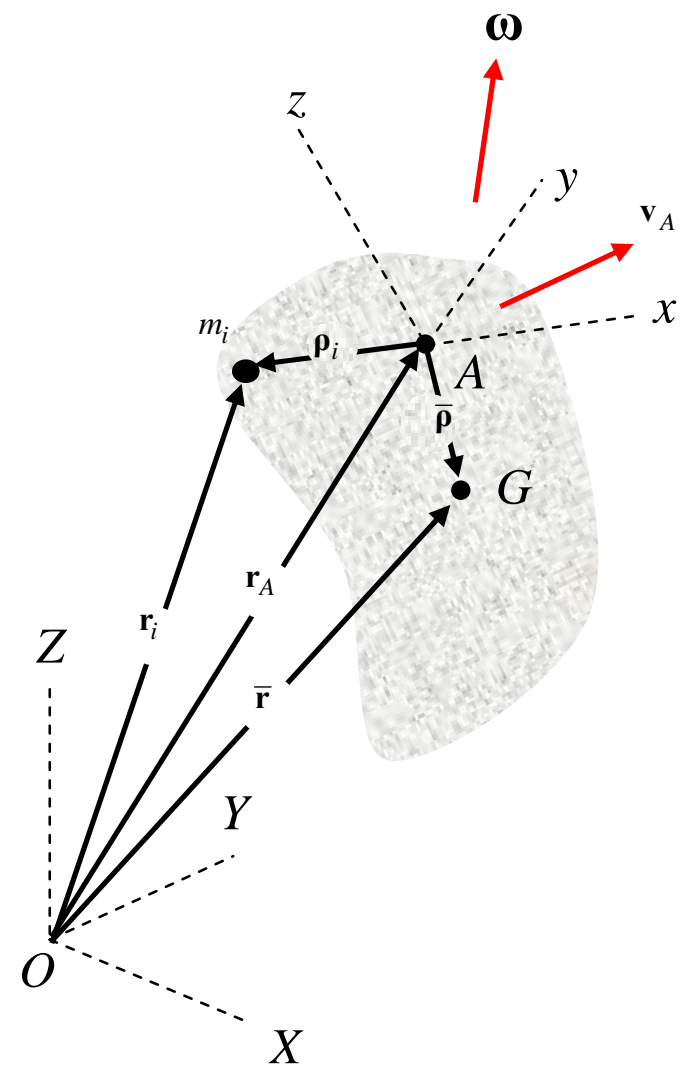

Figure 2. Arbitrary body referenced in inertial and body-centric frames.

\section{A. Translational Acceleration}

Consider the rigid body in figure 2. Let the $X-Y-Z$ reference frame be fixed inertially at point $O$ and the $x-y-Z$ reference frame be attached to the body at any arbitrary point $A$. The body is free to move and has an instantaneous angular rate $\boldsymbol{\omega}$ with respect to reference $X-Y$-Z . Let $m_{i}$ denote a particle of mass of the body located in the fixed inertial reference frame as

$$
\mathbf{r}_{i}=\mathbf{r}_{A}+\boldsymbol{\rho}_{i}
$$

where $\mathbf{r}_{A}$ defines the position of point $A$ with respect to $O$ and $\boldsymbol{\rho}_{i}$ defines the position of $m_{i}$ with respect to point $A$. Furthermore, let $G$ denote the center of mass located by $\overline{\boldsymbol{\rho}}$ from $A$ and $\overline{\mathbf{r}}$ from $O$. From Varignon's Theorem ${ }^{2}, \overline{\boldsymbol{\rho}}$ 
and $\overline{\mathbf{r}}$ satisfy $m \overline{\boldsymbol{\rho}}=\sum m_{i} \boldsymbol{\rho}_{i}$ and $m \overline{\mathbf{r}}=\sum m_{i} \mathbf{r}_{i}$, respectively, where $m$ is the total mass of the body, $m=\sum m_{i}$. From Newton's Second Law, the translational motion of the body is governed by

$$
\sum \mathbf{F}=\sum m_{i}\left(\ddot{\mathbf{r}}_{i}\right)_{X Y Z}=m(\ddot{\mathbf{r}})_{X Y Z}
$$

where $\sum \mathbf{F}$ is the sum of all external forces on $m$ and $(\ddot{\overline{\mathbf{r}}})_{X Y Z}$ is the acceleration of the center of mass observed from the fixed inertial reference frame.

To rewrite (2) in terms of components measured relative to the body-fixed $x-y-z$ reference at $A$, take the derivative of (1) with respect to $X-Y-Z$ following the rule

$$
\left(\frac{d[]}{d t}\right)_{X Y Z}=\left(\frac{d[]}{d t}\right)_{x y z}+\boldsymbol{\omega} \times[] \text { so }\left(\dot{\mathbf{r}}_{i}\right)_{X Y Z}=\left(\dot{\mathbf{r}}_{A}\right)_{X Y Z}+\left(\dot{\boldsymbol{\rho}}_{i}\right)_{X Y Z} \text { becomes }
$$

$$
\left(\dot{\mathbf{r}}_{i}\right)_{X Y Z}=\mathbf{v}_{A}+\dot{\boldsymbol{\rho}}_{i}+\boldsymbol{\omega} \times \boldsymbol{\rho}_{i}
$$

where $\mathbf{v}_{A}$ is $\left(\dot{\mathbf{r}}_{A}\right)_{X Y Z}$ expressed in $x-y-Z$ coordinates. Here, $\boldsymbol{\rho}_{i}$ and $\boldsymbol{\omega}$ are expressed in $x-y-Z$ components and $\dot{\boldsymbol{\rho}}_{i}$ is the velocity of $m_{i}$ as observed in the $x-y-z$ frame. To promote economy of notation, ( $)_{x y z}$ will be dropped when the derivative is with respect to the $x-y-z$ frame. Taking the derivative of (3) again with respect to $X$ - $Y$ - $Z$ yields

$$
\left(\ddot{\mathbf{r}}_{i}\right)_{X Y Z}=\left(\frac{d}{d t}\left(\mathbf{v}_{A}+\dot{\boldsymbol{\rho}}_{i}+\boldsymbol{\omega} \times \boldsymbol{\rho}_{i}\right)\right)_{x y z}+\boldsymbol{\omega} \times\left(\mathbf{v}_{A}+\dot{\boldsymbol{\rho}}_{i}+\boldsymbol{\omega} \times \boldsymbol{\rho}_{i}\right)
$$

or

$$
\left(\ddot{\mathbf{r}}_{i}\right)_{X Y Z}=\dot{\mathbf{v}}_{A}+\boldsymbol{\omega} \times \mathbf{v}_{A}+\ddot{\boldsymbol{\rho}}_{i}+\dot{\boldsymbol{\omega}} \times \boldsymbol{\rho}_{i}+2 \cdot\left(\boldsymbol{\omega} \times \dot{\boldsymbol{\rho}}_{i}\right)+\boldsymbol{\omega} \times\left(\boldsymbol{\omega} \times \boldsymbol{\rho}_{i}\right)
$$

Let $\sum \mathbf{F}$ be expressed in components measured relative to the body fixed axis, then

$$
\begin{gathered}
\sum \mathbf{F}=\sum m_{i}\left(\dot{\mathbf{v}}_{A}+\boldsymbol{\omega} \times \mathbf{v}_{A}+\ddot{\boldsymbol{\rho}}_{i}+\dot{\boldsymbol{\omega}} \times \boldsymbol{\rho}_{i}+2 \cdot\left(\boldsymbol{\omega} \times \dot{\boldsymbol{\rho}}_{i}\right)+\boldsymbol{\omega} \times\left(\boldsymbol{\omega} \times \boldsymbol{\rho}_{i}\right)\right) \\
\sum \mathbf{F}=m\left(\dot{\mathbf{v}}_{A}+\boldsymbol{\omega} \times \mathbf{v}_{A}\right)+\sum m_{i} \ddot{\boldsymbol{\rho}}_{i}+2 \cdot\left(\boldsymbol{\omega} \times \sum m_{i} \dot{\boldsymbol{\rho}}_{i}\right)+\dot{\boldsymbol{\omega}} \times \sum m_{i} \boldsymbol{\rho}_{i}+\boldsymbol{\omega} \times\left(\boldsymbol{\omega} \times \sum m_{i} \boldsymbol{\rho}_{i}\right) \\
\sum \mathbf{F}=m\left(\dot{\mathbf{v}}_{A}+\boldsymbol{\omega} \times \mathbf{v}_{A}\right)+m \ddot{\overline{\boldsymbol{\rho}}}+2 \cdot(\boldsymbol{\omega} \times m \dot{\overline{\boldsymbol{\rho}}})+\dot{\boldsymbol{\omega}} \times m \overline{\boldsymbol{\rho}}+\boldsymbol{\omega} \times(\boldsymbol{\omega} \times m \overline{\boldsymbol{\rho}}) .
\end{gathered}
$$

Since the body is rigid $\ddot{\overline{\boldsymbol{\rho}}}=\dot{\overline{\boldsymbol{\rho}}}=0$,

$$
\sum \mathbf{F}=m\left(\dot{\mathbf{v}}_{A}+\boldsymbol{\omega} \times \mathbf{v}_{A}\right)+\dot{\boldsymbol{\omega}} \times m \overline{\boldsymbol{\rho}}+\boldsymbol{\omega} \times(\boldsymbol{\omega} \times m \overline{\boldsymbol{\rho}}) .
$$

To formulate aircraft equations of motion for a non-center of mass reference, define the angular velocity, position of the center of mass, and the velocity at $A$ in the body fixed axis $\mathrm{x}-\mathrm{y}-\mathrm{z}$ as

$$
\begin{aligned}
& \boldsymbol{\omega}=P \mathbf{i}+Q \mathbf{j}+R \mathbf{k} \\
& \overline{\boldsymbol{\rho}}=\Delta x \mathbf{i}+\Delta y \mathbf{j}+\Delta z \mathbf{k} \\
& \mathbf{v}_{A}=U_{A} \mathbf{i}+V_{A} \mathbf{j}+W_{A} \mathbf{k}
\end{aligned}
$$

respectively. Here, the velocity components are subscripted with $A$ to distinguish them from the usual velocity components considered at the center of mass. Of course these components are the velocity of the center of mass when $A$ is the center of mass, $\overline{\boldsymbol{\rho}}=0$. Separating the force of gravity 


$$
\mathbf{W}=-m g \sin \Theta \mathbf{i}+m g \cos \Theta \sin \Phi \mathbf{j}+m g \cos \Theta \cos \Phi \mathbf{k})
$$

from the other external forces, the force driven aircraft equations of motion referenced to an arbitrary body fixed point $A$ are

$$
\begin{aligned}
\sum \mathbf{F}_{X}=m\left(\dot{U}_{A}+Q W_{A}-R V_{A}-\right. & \left.\left(Q^{2}+R^{2}\right) \Delta x+(Q P-\dot{R}) \Delta y+(R P+\dot{Q}) \Delta z+g \sin \Theta\right) \\
\sum \mathbf{F}_{Y}=m\left(\dot{V}_{A}+R U_{A}-P W_{A}\right. & +(P Q+\dot{R}) \Delta x \\
& \left.-\left(P^{2}+R^{2}\right) \Delta y+(Q R-\dot{P}) \Delta z-g \cos \Theta \sin \Phi\right) \\
\sum \mathbf{F}_{Z}=m\left(\dot{W}_{A}+P V_{A}-Q U_{A}\right. & +(P R-\dot{Q}) \Delta x \\
& \left.+(Q R+\dot{P}) \Delta y-\left(P^{2}+Q^{2}\right) \Delta z-g \cos \Theta \cos \Phi\right) .
\end{aligned}
$$

Here, $\Phi$ and $\Theta$ are the standard roll and pitch Euler angles from the standard aircraft yaw-pitch-roll Euler rotation sequence defining the bodies orientation to the fixed inertial reference frame. ${ }^{3}$ The angular acceleration terms in (10-12) and additional angular rate terms are due to the center of mass offset from the body fixed frame. Solution of the coupled translational and angular accelerations will be discussed after the equations governing the angular rates are formulated for the offset body fixed axes.

\section{B. Angular Acceleration}

Consider again the rigid body described in figure 2 where the $X-Y-Z$ reference frame is fixed inertially at point $O$ and $x-y-Z$ reference is attached to body at any arbitrary point $A$. The body is free to move and has an instantaneous angular velocity, $\boldsymbol{\omega}$, observed from the $X-Y-Z$ reference frame. Again, let $m_{i}$ denote a particle of mass of the body located from the fixed frame as

$$
\mathbf{r}_{i}=\mathbf{r}_{\mathrm{A}}+\boldsymbol{\rho}_{i}
$$

where $\mathbf{r}_{A}$ defines the position of point $A$ with respect to $O$ and $\boldsymbol{\rho}_{i}$ defines the position of $m_{i}$ with respect to point $A$. In this section, the momentum principle, relating the rate change of angular momentum to the external moments, is formulated for the moment of linear momenta taken about $A$.

The absolute angular moment about $A$ is defined as

$$
\mathbf{H}_{A}=\sum\left(\boldsymbol{\rho}_{i} \times m_{i} \mathbf{v}_{i}\right)
$$

where $\mathbf{v}_{i}=\left(\dot{\mathbf{r}}_{i}\right)_{X Y Z}$ is the velocity of $m_{i}$ observed from the $X-Y-Z$ reference frame. The derivative of $\mathbf{H}_{A}$ observed from the $X-Y-Z$ reference frame is

$$
\left(\dot{\mathbf{H}}_{A}\right)_{X Y Z}=\sum\left(\left(\dot{\boldsymbol{\rho}}_{i}\right)_{X Y Z} \times m_{i} \mathbf{v}_{i}\right)+\sum\left(\boldsymbol{\rho}_{i} \times m_{i}\left(\dot{\mathbf{v}}_{i}\right)_{X Y Z}\right)
$$

where $\left(\dot{\boldsymbol{\rho}}_{i}\right)_{X Y Z}$ and $\left(\dot{\mathbf{v}}_{i}\right)_{X Y Z}$ are derivatives observed from the $X-Y$ - $Z$ reference. From figure $2,\left(\dot{\boldsymbol{p}}_{i}\right)_{X Y Z}=\left(\dot{\mathbf{r}}_{i}-\dot{\mathbf{r}}_{A}\right)_{X Y Z}$ and $\left(\dot{\mathbf{v}}_{i}\right)_{X Y Z}=\left(\ddot{\mathbf{r}}_{i}\right)_{X Y Z}$. Since $\left(\dot{\mathbf{r}}_{i}\right)_{X Y Z} \times \mathbf{v}_{i}=0$ and the sum of external moments about $A$ is defined as $\sum \mathbf{M}_{A}=\sum\left(\boldsymbol{\rho}_{i} \times m_{i}\left(\ddot{\mathbf{r}}_{i}\right)_{X Y Z}\right),{ }^{2}(14)$ can be rewritten as

$$
\sum \mathbf{M}_{A}=\left(\dot{\mathbf{H}}_{A}\right)_{X Y Z}+\left(\dot{\mathbf{r}}_{A}\right)_{X Y Z} \times \sum m_{i} \mathbf{v}_{i}
$$


The velocity of the center of mass with respect to frame $X-Y-Z, \overline{\mathbf{v}}$, satisfies $m \overline{\mathbf{v}}=\sum m_{i} \mathbf{v}_{i}$. Noting $\overline{\mathbf{v}}=\mathbf{v}_{A}+(\dot{\overline{\mathbf{p}}})_{X Y Z}$ where both $\mathbf{v}_{A}\left(=\left(\dot{\mathbf{r}}_{A}\right)_{X Y Z}\right)$ and $(\dot{\overline{\mathbf{p}}})_{X Y Z}$ are observed from $X-Y$-Z, the desired momentum principle equation is

$$
\sum \mathbf{M}_{A}=\left(\dot{\mathbf{H}}_{A}\right)_{X Y Z}+\mathbf{v}_{A} \times m(\dot{\overline{\mathbf{p}}})_{X Y Z} .
$$

If $A$ is the center of mass, $\overline{\mathbf{v}}$ equals $\mathbf{v}_{A}$ and (15) assumes the usual form, $\sum \mathbf{M}_{A}=\left(\dot{\mathbf{H}}_{A}\right)_{X Y Z}$. In order to express (16) in components measured in the body fixed frame $x-y-z$ at $A$, first express (13) as

$$
\mathbf{H}_{A}=\sum\left(\boldsymbol{\rho}_{i} \times m_{i}\left(\mathbf{v}_{A}+\dot{\boldsymbol{\rho}}_{i}+\boldsymbol{\omega} \times \boldsymbol{\rho}_{i}\right)\right)
$$

where $\dot{\boldsymbol{\rho}}_{i}$ is the velocity of $m_{i}$ observed in frame $x-y$-z. Vectors $\dot{\boldsymbol{\rho}}_{i}, \boldsymbol{\rho}_{i}, \boldsymbol{\omega}$, and $\mathbf{v}_{A}$ are all expressed in components measured in the body fixed $x-y-z$ frame. Since the body is rigid, $\dot{\boldsymbol{\rho}}_{i}=0$ so

$$
\begin{gathered}
\mathbf{H}_{A}=\sum m_{i} \boldsymbol{\rho}_{i} \times \mathbf{v}_{A}+\sum \boldsymbol{\rho}_{i} \times m_{i}\left(\boldsymbol{\omega} \times \boldsymbol{\rho}_{i}\right) \text { or } \\
\mathbf{H}_{A}=m \overline{\boldsymbol{\rho}} \times \mathbf{v}_{A}+\mathbf{I} \boldsymbol{\omega}
\end{gathered}
$$

where $\mathbf{I}$ is the mass moment of inertia matrix of the rigid body. $\mathbf{I}$ is defined relative to the body fixed $x-y-z$ at $A$ and hence, is invariant with time, $\dot{\mathbf{I}}=0$. I $\mathbf{\omega}$ is a vector in the $x-y-z$ frame arising from the integration version of $\sum \boldsymbol{\rho}_{i} \times m_{i}\left(\boldsymbol{\omega} \times \boldsymbol{\rho}_{i}\right), \int \boldsymbol{\rho} \times(\boldsymbol{\omega} \times \boldsymbol{\rho}) d m$ and will be given below. Since $\mathbf{H}_{A}$ and $\overline{\boldsymbol{\rho}}$ are now expressed in components measured relative to frame $x-y-z$, the needed absolute derivatives measured from $X-Y-Z$ in (16) are obtained from

$$
\left(\dot{\mathbf{H}}_{A}\right)_{X Y Z}=\left(\dot{\mathbf{H}}_{A}\right)_{x y z}+\boldsymbol{\omega} \times \mathbf{H}_{A}
$$

and

$$
(\dot{\overline{\boldsymbol{\rho}}})_{X Y Z}=(\dot{\overline{\boldsymbol{\rho}}})_{x y z}+\boldsymbol{\omega} \times \overline{\boldsymbol{\rho}} .
$$

Substituting (17) into (18) yields

$$
\begin{gathered}
\left(\dot{\mathbf{H}}_{A}\right)_{X Y Z}=\left(\frac{d}{d t}\left(m \overline{\boldsymbol{\rho}} \times \mathbf{v}_{A}+\mathbf{I} \boldsymbol{\omega}\right)\right)_{X Y Z}+\boldsymbol{\omega} \times\left(m \overline{\boldsymbol{\rho}} \times \mathbf{v}_{A}+\mathbf{I} \boldsymbol{\omega}\right) \\
\left(\dot{\mathbf{H}}_{A}\right)_{X Y Z}=m \dot{\overline{\boldsymbol{\rho}}} \times \mathbf{v}_{A}+m \overline{\boldsymbol{\rho}} \times \dot{\mathbf{v}}_{A}+\mathbf{I} \dot{\boldsymbol{\omega}}+\boldsymbol{\omega} \times\left(m \overline{\boldsymbol{\rho}} \times \mathbf{v}_{A}\right)+\boldsymbol{\omega} \times \mathbf{I} \boldsymbol{\omega} \\
\left(\dot{\mathbf{H}}_{A}\right)_{X Y Z}=\mathbf{I} \dot{\boldsymbol{\omega}}+\boldsymbol{\omega} \times \mathbf{I} \boldsymbol{\omega}+m \overline{\boldsymbol{\rho}} \times \dot{\mathbf{v}}_{A}+\boldsymbol{\omega} \times\left(m \overline{\boldsymbol{\rho}} \times \mathbf{v}_{A}\right) .
\end{gathered}
$$

As before, derivatives with no noted referenced axes are assumed to be with respect to the body fixed $x-y-z$ frame, i.e. ( $)_{x y z}$. With regard to (19), the body is rigid, thus, the center of mass does not move relative to A. So $\dot{\overline{\boldsymbol{\rho}}}=0$ and $(\dot{\overline{\boldsymbol{\rho}}})_{X Y Z}=\boldsymbol{\omega} \times \overline{\boldsymbol{\rho}}$. Substituting this result and (20) into (16) yields

$$
\sum \mathbf{M}_{A}=\mathbf{I} \dot{\boldsymbol{\omega}}+\boldsymbol{\omega} \times \mathbf{I} \boldsymbol{\omega}+m \overline{\boldsymbol{\rho}} \times \dot{\mathbf{v}}_{A}+m \boldsymbol{\omega} \times\left(\overline{\boldsymbol{\rho}} \times \mathbf{v}_{A}\right)+m \mathbf{v}_{A} \times(\boldsymbol{\omega} \times \overline{\boldsymbol{\rho}}) .
$$


Redefining $\sum \mathbf{M}_{A}$ to not include the moment at $A$ due to the gravity force acting through the center of mass yields

$$
\sum \mathbf{M}_{A}=\mathbf{I} \dot{\boldsymbol{\omega}}+\boldsymbol{\omega} \times \mathbf{I} \boldsymbol{\omega}+m \overline{\boldsymbol{\rho}} \times \dot{\mathbf{v}}_{A}+m \boldsymbol{\omega} \times\left(\overline{\boldsymbol{\rho}} \times \mathbf{v}_{A}\right)+m \mathbf{v}_{A} \times(\boldsymbol{\omega} \times \overline{\boldsymbol{\rho}})-\overline{\boldsymbol{\rho}} \times \mathbf{W},
$$

the desired moment equations of motion referenced to the arbitrary body fixed point $A$.

For the moment driven aircraft equations of motion, note $\mathbf{I} \boldsymbol{\omega}$ is shorthand for

$$
\mathbf{I} \boldsymbol{\omega}=\left(I_{x x} P-I_{x y} Q-I_{x z} R\right) \mathbf{i}+\left(-I_{x y} P+I_{y y} Q-I_{y z} R\right) \mathbf{j}+\left(-I_{x z} P-I_{y z} Q+I_{z z} R\right) \mathbf{k}
$$

with no assumptions of symmetry about the $x$-z plane (i.e., $I_{y z} \neq 0, I_{x y} \neq 0$ ). So (22) expands to

$$
\begin{aligned}
\sum \mathbf{M}_{A_{x}}=I_{x x} \dot{P}-I_{x y} \dot{Q}-I_{x z} \dot{R}+I_{x y} P R-I_{x z} P Q+\left(I_{z z}-I_{y y}\right) Q R+\left(R^{2}-Q^{2}\right) I_{y z} & \\
& +m\left(\left(P V_{A}-Q U_{A}+\dot{W}_{A}-g \cos \Theta \cos \Phi\right) \Delta y+\left(P W_{A}-R U_{A}-\dot{V}_{A}+g \cos \Theta \sin \Phi\right) \Delta z\right) \\
\sum \mathbf{M}_{A_{y}}=-I_{x y} \dot{P}+I_{y y} \dot{Q}-I_{y z} \dot{R}+I_{y z} P Q-I_{x y} Q R+\left(I_{x x}-I_{z z}\right) P R+\left(P^{2}-R^{2}\right) I_{x z} & \\
+ & m\left(\left(Q U_{A}-P V_{A}-\dot{W}_{A}+g \cos \Theta \cos \Phi\right) \Delta x+\left(Q W_{A}-R V_{A}+\dot{U}_{A}+g \sin \Theta\right) \Delta z\right) \\
\sum \mathbf{M}_{A_{z}}=-I_{x z} \dot{P}-I_{y z} \dot{Q}+I_{z z} \dot{R}+I_{x z} Q R-I_{y z} P R+\left(I_{y y}-I_{x x}\right) P Q+\left(Q^{2}-P^{2}\right) I_{x y} & +m\left(\left(R U_{A}-P W_{A}+\dot{V}_{A}-g \cos \Theta \sin \Phi\right) \Delta x+\left(R V_{A}-Q W_{A}-\dot{U}_{A}-g \sin \Theta\right) \Delta y\right)
\end{aligned}
$$

As with the force equations, the moment equations contain coupling of both angular and translational accelerations due to the center of mass offset.

\section{Modeling Large Instantaneous Center of Mass Shift}

In the previous section, equations of motion were developed referenced to a point fixed on a rigid body, not necessarily the center of mass, with the center of mass fixed relative to this point. The problem of interest, however, is not one where the center of mass is fixed but one where at some point in time the center of mass instantaneously shifts to another location on the body. Consider a rigid body that at some time a piece of the body breaks away and motion of the remaining piece is of interest. Without loss of generality, assume that reference point A, discussed above, is attached to the body of interest. Examples include a fighter aircraft releasing its stores, a free falling space capsule separating from its launch abort tower, and an aircraft losing part of its wing. Momentum exchange due to mid-air collisions resulting in wing loss is not modeled here but can be included in the form of transient forces and moments.

In this section, we contrast a more traditional center of mass (CM)-centric approach with one utilizing the equations developed above. The simulation modified for this study was of a subscale generic transport model that ran with fixed-time step, $\Delta T$, Euler integration. Here, assume the center of mass shift occurs at some multiple of the time step, e.g. $t=k \Delta T$.

\section{A. CM-Centric Approach}

The problem considered should not be confused with describing the motion of a rigid body with incremental changes in center of mass, e.g. fuel is burned. The general practice for incremental center of mass change is at each time step transfer the moments due to external forces referenced to some body fixed point to the new center of mass. The mass is modified along with the inertia tensor written with respect to the new center of mass, generally using the parallel axis theorem on constant mass subsystems. If the center of mass change is small, at each time step, the velocity at the center of mass of the previous time step is close enough to the velocity at the new center of mass so

6 of 13 
the integration can run with that as the initial condition for the next integration. When the center of mass change is large, however, the velocity corresponding to the previous center of mass is appropriate as the next step initial condition for integration only when angular rate is near zero. If the body is experiencing any angular rate, as is common in aircraft maneuvering, such a strategy leads to poorly modeled transients since the initial condition does not reflect the velocity at the new center of mass.

In the event of a large instantaneous change in center of mass, a carefully choreographed reset (initial condition change) of the integrators corresponding to $\dot{\overline{\mathbf{v}}}$ must occur. This reset involves 1 ) a trigger which detects the center of mass shift, 2) a calculation of $\overline{\mathbf{v}}$ and $\dot{\overline{\mathbf{v}}}$ of the new center of mass location based on the center of mass shift and on previous values of the state and state rates of the previous center of mass location, 3 ) a prediction of the current $\overline{\mathbf{v}}$ of the new center of mass location utilizing the results of 2), and 4) a reset of the integrator's initial condition using this $\overline{\mathbf{v}}$ of the new center of mass triggered by 1). The equations governing the motion of the center of mass can be obtained above by setting the center of mass offset from $A$ to zero. Note, $\boldsymbol{\omega}$ and $\dot{\boldsymbol{\omega}}$ (as well as the Euler angles and rates) correspond to the rigid body, not specific to any fixed point on the body. As a result, the integrators on $\dot{\boldsymbol{\omega}}$ need not be reset.

In the simulation, the state $[\overline{\mathbf{v}}(k \Delta T), \boldsymbol{\omega}(k \Delta T)]$ and the previous value of its rate $[\dot{\overline{\mathbf{v}}}((k-1) \Delta T)$, $\dot{\boldsymbol{\omega}}((k-1) \Delta T)]$ are fed back to an auxiliary subsystem for defining angle of attack, sideslip, and their rates required as independent variables in the definition of aerodynamic forces and moments. Strictly speaking, these signals are used to determine the motion of the body-fixed aerodynamic reference point (defined in the wind tunnel test) as an appropriate definition of angle of attack and sideslip. In Euler integration, the held rates are the one-sided state derivatives at $t=k \Delta T$ approaching from left side. In this paper, both state and the previous rate will be considered current values and denoted as $\overline{\mathbf{v}}_{c m 1}^{k}$ and $\dot{\overline{\mathbf{v}}}_{c m 1}^{k}$. Here subscripts $c m 0$ and $c m 1$ denote its previous and new center of mass locations, respectively. Let $\overline{\mathbf{r}}_{c m 0}^{k-1}$ and $\overline{\mathbf{r}}_{c m 1}^{k}$ define the position of the center of mass for each location, and $\Delta \overline{\mathbf{r}}=\overline{\mathbf{r}}_{c m 1}^{k}-\overline{\mathbf{r}}_{c m 0}^{k-1}$ define the change in center of mass with respect to its previous location. The center of mass location is generally determined in the mass and inertia subsystem of the simulation. The trigger detects a rise in norm of $\Delta \overline{\mathbf{r}}$ at $t=k \Delta T$. To construct the state at $c m 1$ at $t=k \Delta T$, previous values of the state and its rate defined at $\mathrm{cm} 0$ are used as follows. First the previous state and rate at $\mathrm{cm} 1$ are constructed

$$
\begin{aligned}
\overline{\mathbf{v}}_{c m 1}^{k-1} & =\overline{\mathbf{v}}_{c m 0}^{k-1}+\boldsymbol{\omega}^{k-1} \times \Delta \overline{\mathbf{r}} \\
\dot{\overline{\mathbf{v}}}_{c m 1}^{k-1} & =\dot{\overline{\mathbf{v}}}_{c m 0}^{k-1}+\dot{\boldsymbol{\omega}}^{k-1} \times \Delta \overline{\mathbf{r}} .
\end{aligned}
$$

An Euler prediction provides an estimate of the current state at $c g 1$ at $t=k \Delta T$.

$$
\overline{\mathbf{v}}_{c m 1}^{k} \cong \overline{\mathbf{v}}_{c m 1}^{k-1}+\dot{\overline{\mathbf{v}}}_{c m 1}^{k-1} \Delta T
$$

This estimate is used as an initial condition to reset the integrators of the translational state rates at $t=k \Delta T$. The non-CM approach using the equations of the previous section avoids this reset problem entirely because reference point $A$ is stationary on the body.

\section{B. Non-CM Approach}

The non-CM approach does not imply that the location of the center of mass is not needed. The location of the center of mass with respect to $A, \overline{\boldsymbol{\rho}}$, is required in the equations of motion. Let the forces and moments due to gravity be subsumed back into the external force and moment, $\sum \mathbf{F}$ and $\sum \mathbf{M}_{A}$, respectively. Equations (10-12) \& (24-26) can be concisely expressed in matrix notation

$$
\left[\begin{array}{c}
\dot{\mathbf{v}}_{A} \\
\dot{\boldsymbol{\omega}}
\end{array}\right]=\left[\begin{array}{cc}
m \mathbf{I}_{3} & -\mathbf{D}_{x} \\
\mathbf{D}_{x} & \mathbf{I}
\end{array}\right]^{-1}\left[\left[\begin{array}{c}
\sum \mathbf{F} \\
\sum \mathbf{M}_{A}
\end{array}\right]-\left[\begin{array}{cc}
m \boldsymbol{\Omega}_{x} & -\boldsymbol{\Omega}_{x} \mathbf{D}_{x} \\
\boldsymbol{\Omega}_{x} \mathbf{D}_{x} & \boldsymbol{\Omega}_{x} \mathbf{I}-\mathbf{V}_{x} \mathbf{D}_{x}
\end{array}\right]\left[\begin{array}{c}
\mathbf{v}_{A} \\
\boldsymbol{\omega}
\end{array}\right]\right]
$$

7 of 13 
where

$$
\begin{aligned}
& \mathbf{D}_{x}=\left[\begin{array}{ccc}
0 & -m \Delta z & m \Delta y \\
m \Delta z & 0 & -m \Delta x \\
-m \Delta y & m \Delta x & 0
\end{array}\right], \boldsymbol{\Omega}_{x}=\left[\begin{array}{ccc}
0 & -R & Q \\
R & 0 & -P \\
-Q & P & 0
\end{array}\right] \\
& \mathbf{V}_{x}=\left[\begin{array}{ccc}
0 & -W_{A} & V_{A} \\
W_{A} & 0 & -U_{A} \\
-V_{A} & U_{A} & 0
\end{array}\right], \text { and } \mathbf{I}=\left[\begin{array}{ccc}
I_{x x} & -I_{x y} & -I_{x z} \\
-I_{x y} & I_{y y} & -I_{y z} \\
-I_{x z} & -I_{y z} & I_{z z}
\end{array}\right] .
\end{aligned}
$$

Here, the inertia matrix is expressed about fixed point reference $A$. If part of the wing of an aircraft breaks off at $t=k \Delta T$ with mass $m_{\text {tip }}$ and center of mass located with respect to $A$ at $\overline{\boldsymbol{\rho}}_{\text {tip }}$, i.e. $\left(\Delta x_{\text {tip }}, \Delta y_{\text {tip }}, \Delta z_{\text {tip }}\right)$, and an inertia matrix defined about the tip's center of mass, $\mathbf{I}_{t i p, c g}$, the damaged aircraft (dac)'s mass, center of mass, and inertia matrix replace the nominal as

$$
\begin{gathered}
m \rightarrow m_{d a c}=m-m_{t i p} \\
\overline{\boldsymbol{\rho}} \rightarrow \overline{\boldsymbol{\rho}}_{d a c}=m \overline{\boldsymbol{\rho}}-m_{t i p} \overline{\boldsymbol{\rho}}_{t i p} / m_{d a c} \\
I_{i j} \rightarrow I_{i j, d a c}=I_{i j}-I_{i j, t i p} \quad i, j=x, y, z \\
I_{i i, t i p}=I_{i i, t i p, c m}+m_{t i p}\left(\Delta j_{t i p}^{2}+\Delta k_{t i p}^{2}\right) \quad i, j, k=x, y, z \mid i \neq j \& i \neq k \\
I_{i j, t i p}=I_{i j, t i p, c m}+m_{t i p} \Delta i_{t i p} \Delta j_{t i p} \quad i, j=x, y, z \mid i \neq j
\end{gathered}
$$

Here, only the piece removed is subtracted from the inertia matrix. This is in contrast to the CM-centric approach which requires the inertias of both the undamaged vehicle and the missing tip to be transferred to the new center of mass and then subtracted.

Perhaps the only disadvantage in using the non-CM equations of motion is the requirement that a $6 \times 6$ symmetric matrix be inverted to provide the state rates. Computationally this does not seem to be a burden for simulation environments such as SIMULINK. It should be pointed out, however, that the input to the subsystem defining angle of attack and sideslip, as well as other required variables are defined with respect to states and rates at the vehicle's center of mass. This does not pose a problem since these values are easily obtained with

$$
\begin{aligned}
\overline{\mathbf{v}}_{c m 1}^{k} & =\overline{\mathbf{v}}_{A}^{k}+\boldsymbol{\omega}^{k} \times \Delta \overline{\boldsymbol{\rho}}_{d a c} \\
\dot{\overline{\mathbf{v}}}_{c m 1}^{k} & =\dot{\overline{\mathbf{v}}}_{A}^{k}+\dot{\boldsymbol{\omega}}^{k} \times \Delta \overline{\boldsymbol{\rho}}_{d a c} .
\end{aligned}
$$

These calculations are performed upstream from the auxiliary and aerodynamic subsystems that need them. Furthermore the auxiliary system defining angle of attack and sideslip can be greatly simplified by choosing the aerodynamic reference center as $A$, since each is defined by the velocity of the aerodynamic reference.

Other benefits of the non-CM approach include the following. Only one moment arm needs to be monitored-that corresponding to gravitational force applied at the center of mass. Assuming the wind tunnel data has been specified at some aerodynamic reference fixed to the vehicle, the moment arm from fixed point reference $A$ to the aerodynamic reference is time invariant-ideally zero with the correct selection of $A$. Since the reference $A$ is body fixed during the damage event, the velocity states at $A$ provide the appropriate initial state for the next integration step. No reset of the integrator is needed! There is no need for the trigger logic. There is no need for predicting the 
current state at the new center of mass from previous states and rates corresponding to the previous center of mass to obtain the appropriate initial condition reset in a timely manner. In the next section, an example will illustrate that the non-CM approach duplicates the results of the CM-centric approach.

\section{Example: Dynamic Response of Generic Transport Model with Damage}

Wind tunnel test for several cases of damage was conducted in 2006 at NASA Langley 14x22 Subsonic Tunnel. Figure 3 illustrates different damage cases tested. Both static and dynamic aerodynamic data along with aerodynamic increments for damage are incorporated into the Generic Transport Model (GTM) simulation along with corresponding changes in aircraft weight and inertia. In this example, the aircraft is trimmed at level flight. At five seconds, the aircraft responds to a 1.5 deg aileron command to roll right for 5 seconds. At ten seconds, the roll rate is zeroed for the next 5 seconds, and then at 15 seconds, wings are leveled with a 1.5 deg. aileron command to roll left for 5 seconds. Prior to completing this maneuver at 19 seconds, $24 \%$ of the left wing's semi-span breaks off with mass equal to $1.95 \%$ of the entire vehicle.

The mass of the missing piece may not be representative of actually losing $24 \%$ of the left wing. The GTM is a 5.5\% dynamically scaled version of a general full-scale transport. A CAD generated model of the missing piece incorporating ribs, stringers, spars, and skin for the full-scale aircraft was constructed. To obtain the subscale version, similitude ${ }^{4}$ rules dictate that the mass and inertia of the full-scale vehicle be multiplied by $(.055)^{3}$ and $(.055)^{5}$, respectively. To provide a $1.95 \%$ drop in the vehicle's mass, a factor was applied to the density of the material used in the full scale model. This factor modified both the missing piece's mass and inertias. For a vehicle with a $6.8488 \mathrm{ft}$ wingspan, this center of mass shift was 0.041 inch forward to nose, 0.715 inch to the right wing, and 0.052 inch up from the nominal position — not a lot of change, but enough to show both approaches yield the same result. To clearly illustrate differences in motion of the two reference points considered, reference $A$ was arbitrarily selected to be 2.0 inches behind, 4.8 inches to the right, and 4.5 inches above the nominal center of mass.

Figure 4 shows the results for the CM-centric approach in blue and non-CM approach in red for the translational velocities coming from the integrator on the left side and the velocities at the center of mass generated by both approaches on the right side. As expected the velocities at reference $A$ differ from those at the center of mass, but the center-of-mass velocities calculated from the reference $A$ states (i.e. equations (37-38)) overlay the CM-centric results.

A closer look at the instant of failure, in figure 5, reveals that the Non-CM approach exactly captures the velocity of the $\mathrm{CM}$ at upset without the need to reset the integrator! The angular rates and Euler angles are shown in figure 6 also reveal duplicate results.

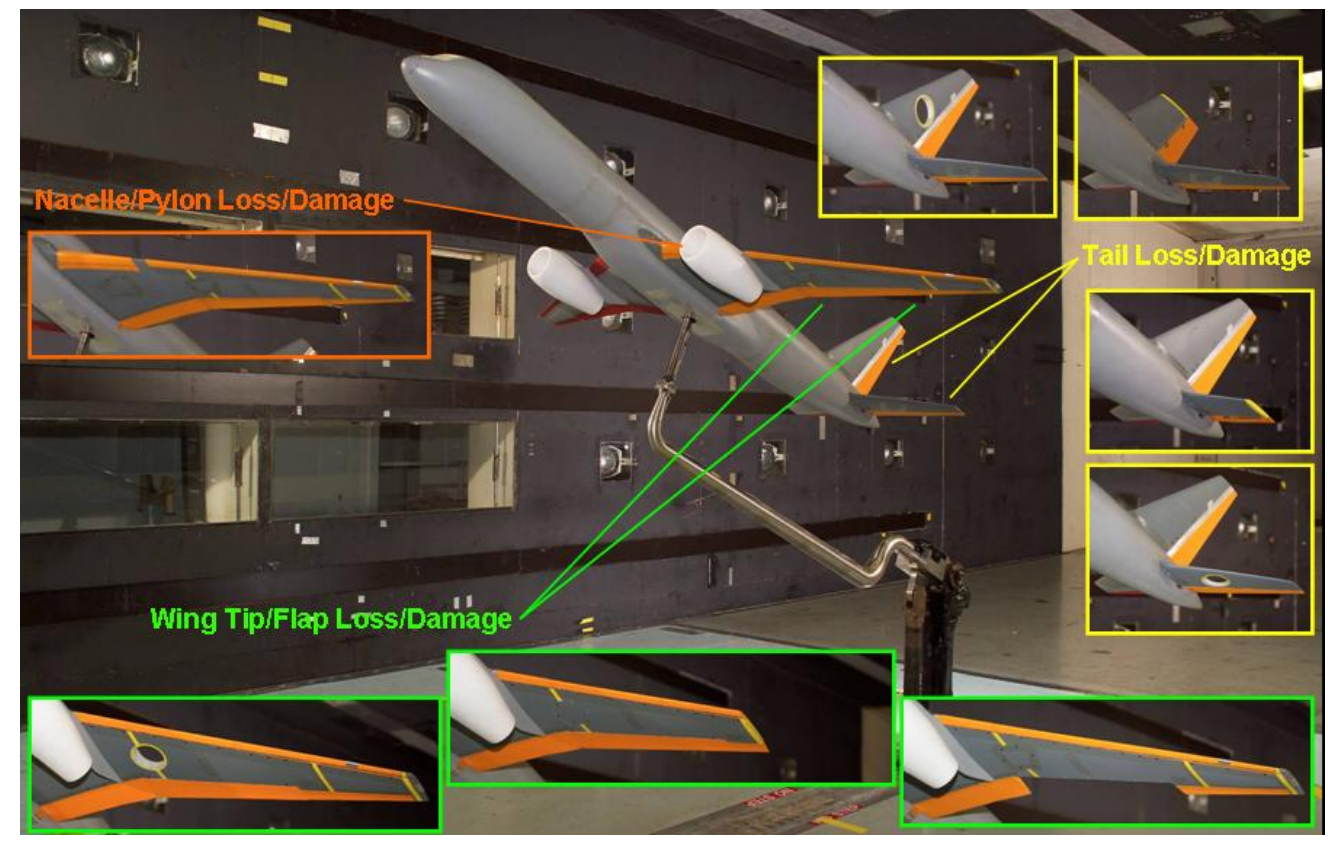

Figure 3. Commercial transport damage research .

9 of 13 

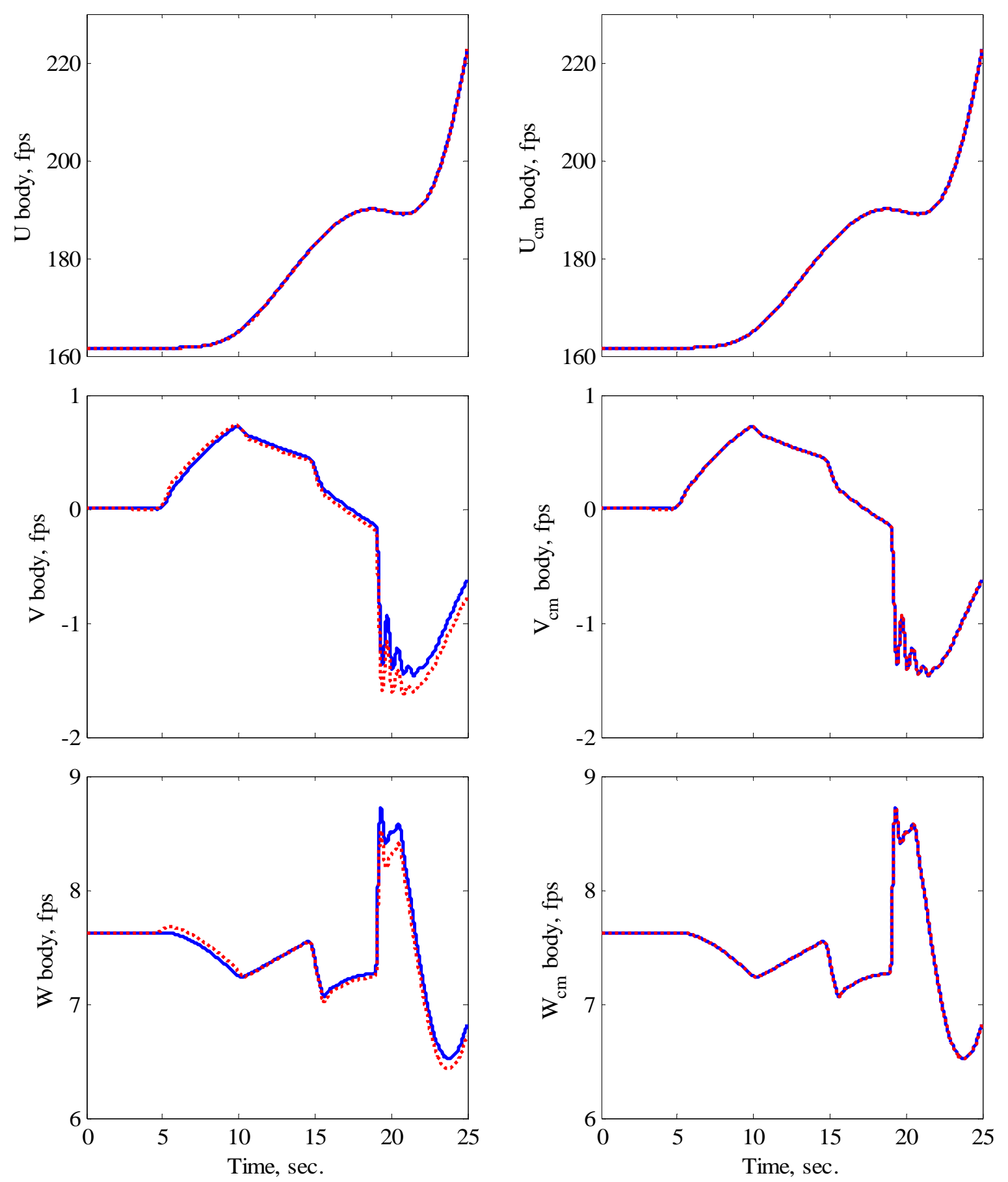

Figure 4. U, V, W from integrator for CM-centric (blue) and non-CM approach (red), left, and calculated at center of mass, right. 

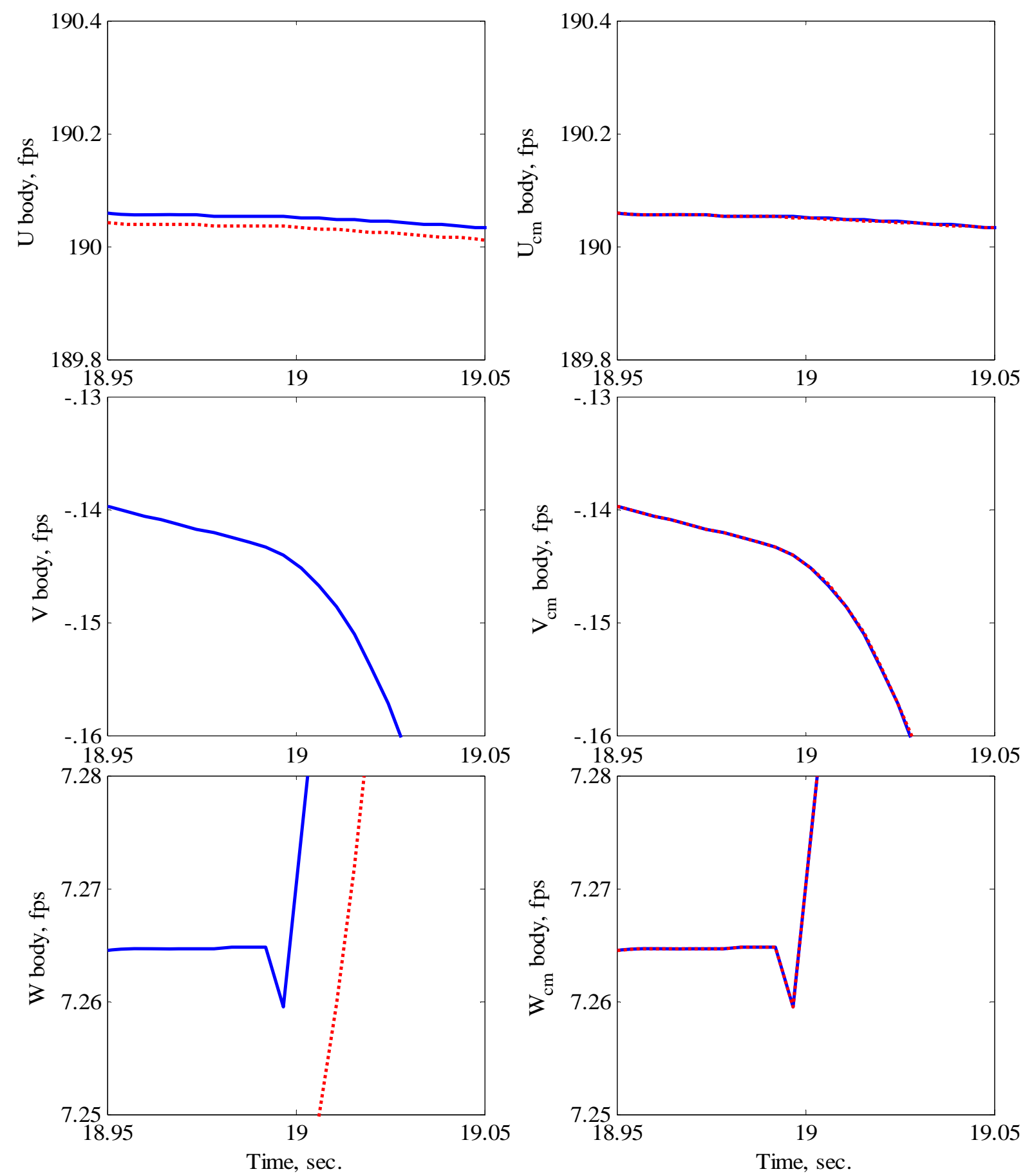

Figure 5. Closer view at failure of $\mathrm{U}, \mathrm{V}, \mathrm{W}$ from integrator for CM-centric (blue) and non-CM approach (red), left, and calculated at center of mass, right. 

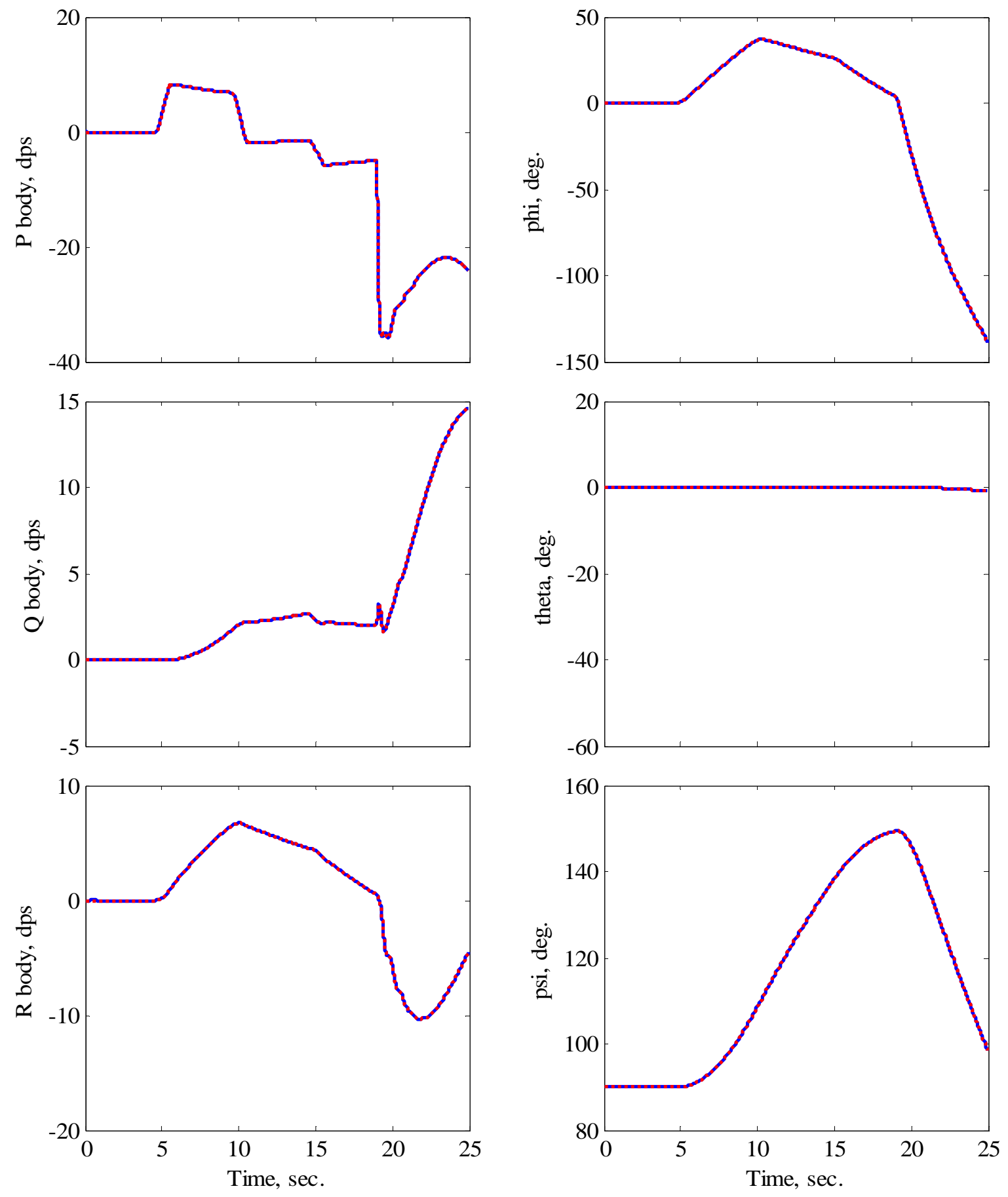

Figure 6. Angular rate and Euler angle comparison

\section{Conclusion}

The Non-CM approach for modeling a rigid body's motion through an instantaneous shift in the body's center of mass as a result of mass loss essentially duplicates the result of the more standard CM-centric approach to simulation. The Non-CM approach utilizes equations of motion expressed about a fixed point on the rigid body that 
does not have to be the center of mass. If the body has any appreciable level of angular rate at the instant that a piece breaks off, the standard CM-centric approach must reset the integrator on $\dot{\overline{\mathbf{v}}}$ with the initial condition of $\overline{\mathbf{v}}$ at the new center of mass location. This discontinuity is avoided entirely with the Non-CM approach, since reference $A$ is fixed to the same point before and after the damage event. As a result the Non-CM approach does not require an integrator reset and all the numerical manipulation that involves: trigger detection, calculation of past motion states of the new center of mass prior to damage, and prediction of the current motion states at the new center of mass required to reset the integral's initial condition.

The Non-CM approach does require tracking of the body's center of mass as does the CM-centric approach. The center of mass position appears in the Non-CM equations of motion and is required for solving the moment due to gravitational force. However, this is the only moment arm that changes. Both aerodynamic and propulsive forces and moments are generally written with respect to a fixed point on the vehicle. Since reference $A$ is also fixed, the moment arms used to translate these forces and moments to reference $A$ are time invariant and need only be calculated at the initiation of the simulation. The inertia matrix, defined about $A$, is easily modified without application of the parallel axis theorem to transfer the undamaged aircraft inertia to the new center of mass. Only the relative distance to the center of mass of the missing piece to $A$, its mass, and inertia of the missing piece defined about its own center of mass are needed to appropriately modify the inertia matrix to that of damaged aircraft about reference $A$. The new center of mass position is determined by the mass and center of mass locations of the undamaged aircraft and the missing wing tip.

The Non-CM approach does come with a price. Instead of inverting a 3x3 inertia matrix, a 6x6 mass-inertia matrix of the coupled system must be inverted. Utilizing the matrix/vector formulation of Section III, SIMULINK, and computational environment available today, this does not appear to be a limiting factor. It took longer for this author to implement a correct integrator reset than to implement the equations of motion of the Non-CM approach.

\section{Acknowledgments}

The authors would like to thank their colleagues from the Dynamic Systems and Control Branch, the Flight Dynamics Branch, and the Durability, Damage Tolerance and Reliability Branch for their time and patience reviewing the equations of motion presented in this paper. Any deficiencies in this paper are authors' own.

\section{References}

${ }^{1}$ Keen, K.S., “Equations for Store Separation Motion Simulations and Instrumented Model Data Reduction.” AEDC-TR-9512, Arnold Engineering Development Center. 1996.

${ }^{2}$ Meriam, J. L. Engineering Mechanics: Dynamics. SI/Eng ed. New York: Wiley, 1978.

${ }^{3}$ McRuer, D., Ashkenas, I., and Graham, D., Aircraft Dynamics and Automatic Control. Princeton, 1973.

${ }^{4}$ Gainer, T.G, and Hoffman, S.. "Summary of Transformation Equations and Equations of Motion used in Free-Flight and Wind-Tunnel Data Reduction and Analysis.” NASA SP-3070, 1972.

13 of 13 